\title{
Soil chemistry determines whether defensive plant secondary metabolites promote or suppress herbivore growth
}

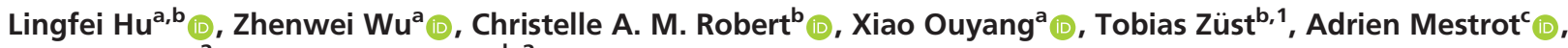 \\ Jianming $\mathrm{Xu}^{\mathrm{a}, 2}$ (D), and Matthias $\mathrm{Erb}^{\mathrm{b}, 2}$ (D) \\ ${ }^{a}$ Institute of Soil and Water Resources and Environmental Science, College of Environmental and Resource Sciences, Zhejiang Provincial Key Laboratory of \\ Agricultural Resources and Environment, Zhejiang University, Hangzhou 310058, China; ${ }^{b}$ Institute of Plant Sciences, University of Bern, 3013 Bern, Switzerland; \\ and 'Institute of Geography, University of Bern, 3012 Bern, Switzerland
}

Edited by James H. Tumlinson, Pennsylvania State University, University Park, PA, and approved September 10, 2021 (received for review May 24, 2021)

Plant secondary (or specialized) metabolites mediate important interactions in both the rhizosphere and the phyllosphere. If and how such compartmentalized functions interact to determine plant-environment interactions is not well understood. Here, we investigated how the dual role of maize benzoxazinoids as leaf defenses and root siderophores shapes the interaction between maize and a major global insect pest, the fall armyworm. We find that benzoxazinoids suppress fall armyworm growth when plants are grown in soils with very low available iron but enhance growth in soils with higher available iron. Manipulation experiments confirm that benzoxazinoids suppress herbivore growth under iron-deficient conditions and in the presence of chelated iron but enhance herbivore growth in the presence of free iron in the growth medium. This reversal of the protective effect of benzoxazinoids is not associated with major changes in plant primary metabolism. Plant defense activation is modulated by the interplay between soil iron and benzoxazinoids but does not explain fall armyworm performance. Instead, increased iron supply to the fall armyworm by benzoxazinoids in the presence of free iron enhances larval performance. This work identifies soil chemistry as a decisive factor for the impact of plant secondary metabolites on herbivore growth. It also demonstrates how the multifunctionality of plant secondary metabolites drives interactions between abiotic and biotic factors, with potential consequences for plant resistance in variable environments.

plant secondary metabolites | benzoxazinoids | herbivore resistance | plant herbivore interactions | maize

0 rganismal traits are commonly coopted for multiple func tions (1 4). In complex and fluctuating environments, multifunctionality may lead to fitness trade offs with important consequences for ecological and evolutionary dynamics (5 7).

Plant secondary (or specialized) metabolites are important mediators of species interactions in natural and agricultural sys tems (8). Many plant secondary metabolites have been docu mented to protect plants against insect herbivores by acting as toxins, digestibility reducers, and/or repellents (9). Plant sec ondary metabolites also serve other functions: they can, for instance, act as signaling molecules (10), photoprotectants (11), antibiotics (12), soil nutrient mobilizers (13), and precursors of primary metabolites (14). Recent genetic work has highlighted that the same plant secondary metabolites may engage in multi ple functions $(4,15,16)$, leading to potentially important inter actions between different environmental factors $(6,17)$. How this multifunctionality influences plant ecology and plant insect interactions in complex environments is not well understood.

The soil environment can have a major impact on plant defense expression and plant herbivore interactions. Soil nutrients and micronutrients can reprogram plant defenses through cross talk between defense and nutrient signaling (18,
$19)$ or by influencing soil microbes, which subsequently modu late plant defense responses $(20,21)$. Elements such as silicon (Si) can also act as defenses directly by forming crystals on the leaf surface (22). In addition, soil nutrients can also influ ence plant herbivore interactions by changing the nutritional value of the plant to herbivores (23). Thus, plant secondary metabolites with dual functions in the rhizosphere and phyllo sphere may mediate interactions between soil chemistry and herbivores (24).

Benzoxazinoids are shikimic acid derived secondary metabo lites that are produced in high abundance by grasses such as wheat and maize. They evolved multiple times within the plant kingdom and are also found in various dicot families (25). Ini tially, benzoxazinoids were described as defense compounds that suppress and repel insect herbivores (26). Later genetic work revealed that benzoxazinoids also act as within plant sig naling compounds by initiating callose deposition upon patho gen and aphid attack $(27,28)$. Benzoxazinoids are also released into the rhizosphere in substantial quantities (29), where they can chelate iron (30), thus making it bioavailable (31). By

\section{Significance}

This study demonstrates that the protective effects of multifunctional maize secondary metabolites against a major pest are dependent on soil chemical composition. By functioning as both digestibility reducers and siderophores, benzoxazinoids link soil chemistry to plant-environment interactions. Given that many plant secondary metabolites have multiple functions in roots and leaves, such links are likely widespread and may govern community composition and pest dynamics across different (agro)ecosystems. The presented findings also illustrate the limits and context dependency of using multifunctional plant secondary metabolites to combat major herbivore pests. The latter is particularly important in the context of the threat that the fall armyworm poses for global maize production.

Author contributions: L.H., C.A.M.R., J.X., and M.E. designed research; L.H., Z.W., X.O., and T.Z. performed research; J.X. and M.E. contributed new reagents/analytic tools; L.H., C.A.M.R., A.M., J.X., and M.E. analyzed data; and L.H., C.A.M.R., and M.E. wrote the paper.

The authors declare no competing interest.

This article is a PNAS Direct Submission.

This open access article is distributed under Creative Commons AttributionNonCommercial-NoDerivatives License 4.0 (CC BY-NC-ND).

${ }^{1}$ Present address: Institute for Systematic and Evolutionary Botany, University of Zürich, 8008 Zürich, Switzerland.

${ }^{2}$ To whom correspondence may be addressed. Email: jmxu@zju.edu.cn or matthias. erb@ips.unibe.ch.

This article contains supporting information online at http://www.pnas.org/lookup/ suppl/doi:10.1073/pnas.2109602118/-/DCSupplemental.

Published October 21, 2021. 
consequence, benzoxazinoids can influence plant iron homeo stasis. Recently, a link was documented between the iron che lating capacity and the interaction between maize plants and the western corn rootworm. This highly adapted insect is attracted by iron benzoxazinoid complexes and can use them for its own iron supply (31). Thus, it is conceivable that the multiple functions of benzoxazinoids may lead to trade offs between their function as defenses and their functions as pro viders of essential micronutrients.

Here, we explore how the multifunctionality of benzoxazi noids shapes interactions between soil conditions and a leaf herbivore. By comparing soils that differ in their trace element composition, we uncover that the protective effect of maize benzoxazinoids against the fall armyworm can be reversed to a susceptibility effect in certain soils. Using micronutrient analy ses and manipulative laboratory experiments, we document that this phenomenon can be explained by the interaction of benzoxa zinoids with free iron in the soil. We further document that iron and benzoxazinoids interact to control leaf defenses but that the benzoxazinoid dependent susceptibility is best explained by increased iron supply to the fall armyworm. Taken together, these results provide a mechanistic link between soil properties and leaf herbivore interactions and illustrate how plant secondary metabolite multifunctionality shapes plant herbivore interactions.

\section{Results}

The Effect of Benzoxazinoids on Herbivore Performance Depends on Soil Type. Benzoxazinoids increase leaf resistance to herbivores but also interact with soil micronutrients and microbial commu nities in the soil (31 33). To test whether the defensive function of benzoxazinoids is modulated by soil properties, we collected soils from eight different arable fields around Yixing (Jiangsu province, China), including anthrosols with expected high avail ability of free iron and ferrosols with expected low availability of iron (classification according to Chinese soil taxonomy, refer to SI Appendix, Fig. S1 and Table S1 for basic soil characteris tics). We then grew wild type B73 (WT) and benzoxazinoid deficient bx1 mutant plants in the different soils, infected the plants with fall armyworm larvae, and measured plant perfor mance, leaf damage, and larval performance. On plants grown in ferrosols, fall armyworm larvae gained more weight on $b x 1$ mutant plants than WT plants, as expected from the defensive function of benzoxazinoids (Fig. 1). However, on plants grown in anthrosols, the larvae gained more weight on WT plants than $b x 1$ mutant plants (Fig. 1). Leaf damage did not differ between genotypes (SI Appendix, Fig. S2A), implying a change in the digestibility of the consumed leaf material. Overall, maize seedlings accumulated more biomass when growing in anthrosols than ferrosols, with no differences between geno types (SI Appendix, Fig. S2B). To confirm that the herbivore growth patterns depend on benzoxazinoid biosynthesis, we tested additional $b x 1$ and $b \times 2$ mutant alleles in the W22 back ground in an anthrosol and a ferrosol. Again, the larvae grew better on $b x 1$ and $b \times 2$ mutants in the ferrosol but grew signifi cantly less on the mutants in the anthrosol (SI Appendix, Fig. $\mathrm{S} 3$ ). Thus, the impact of benzoxazinoid biosynthesis on herbi vore performance is strongly dependent on the soil type.

Soil-Dependent Benzoxazinoid Resistance Is Driven by Root Iron Supply. Benzoxazinoids can chelate micronutrients and trace metals $(30,34)$, with the strongest quenching being observed for iron (31). We thus explored correlations between different soil properties and available micronutrients and the $b x l$ depen dent impact on fall armyworm performance. Principal compo nent analysis (PCA) resulted in a clear separation of anthrosols from ferrosols (Fig. 1I). Ferrosols, on which larvae grew better on $b x 1$ than WT plants, had a slightly higher $\mathrm{pH}$, less organic carbon, very low bioavailable iron, copper, boron, and phospho rus but higher sodium, magnesium, and silicon than anthrosols (Fig. $1 I$ and SI Appendix, Fig. S1). To further narrow down potential micronutrients that may drive the different genotype specific performance of the fall armyworm, we screened micro nutrient levels in the leaves of WT and $b x 1$ plants growing in the different soils. ANOVA revealed significant genotype effects for calcium and iron, with overall higher levels of both elements in the leaves of WT than $b x 1$ mutant plants (SI Appendix, Fig. S4). Furthermore, a significant interaction between genotype and soil type was found for iron (Fig. 1J), with higher iron levels in WT plants than $b x 1$ mutant plants in anthrosols with high available iron and no difference in ferro sols with very low available iron (Fig. $1 J$ ). Overall iron levels were enhanced in plants grown in anthrosols compared to plants grown in ferrosols (Fig. $1 J$ ), which is expected, given that iron in ferrosols is chiefly present as insoluble iron oxide. Quantitative analyses revealed a strong association between higher iron levels in WT plants and higher fall armyworm performance (SI Appendix, Fig. S5). An association was also observed for copper (SI Appendix, Fig. S5), but this pattern was not associated with significant differences in copper levels between genotypes (SI Appendix, Fig. S5). No correlation was observed for calcium (SI Appendix, Fig. S5). The association between plant iron and fall armyworm performance was con served in the W22 background (SI Appendix, Fig. S6).

Based on these results, we hypothesized that differences in iron availability may determine the impact of benzoxazinoids on herbivore performance. To test this hypothesis, we grew WT and $b x 1$ mutant plants in nutrient solutions with different forms of iron (31). Plants where either grown in iron deficient solu tions (supplemented with $\mathrm{NaCl}$ or $\mathrm{Na}_{2} \mathrm{SO}_{4}$ ), solutions contain ing free, soluble iron [supplied as $\mathrm{FeCl}_{3}$ or $\mathrm{Fe}_{2}\left(\mathrm{SO}_{4}\right)_{3}$ ] that requires chelation by siderophores for efficient uptake, or solu tions containing a bioavailable iron complex Fe ethylenediami netetraacetic acid (Fe EDTA). Fall armyworm larvae grew bet ter on benzoxazinoid deficient $b x 1$ mutant plants than WT plants in iron free and Fe EDTA nutrient solutions but worse on $b x 1$ mutant plants than WT plants in solutions containing free iron (Fig. $2 A$ and SI Appendix, Fig. S7). Leaf damage was similar across genotypes and iron treatments (Fig. $2 B$ ). Com plementation of $b x 1$ mutant plants with pure 2,4 dihydroxy 7 methoxy 1,4 benzoxazin 3 one (DIMBOA, $300 \mu \mathrm{g}$ ) added to the rhizosphere reverted the $b x 1$ phenotype in solutions con taining free iron (Fig. 2C). These results show that the interac tion between benzoxazinoids and iron availability can modulate the impact of benzoxazinoids on leaf herbivore performance.

Interactions between Root Iron Supply and Benzoxazinoids Determine Leaf Iron Homeostasis. BxI mutant plants at the seed ling stage are less efficient at taking up free iron than WT plants due to the absence of DIMBOA in the rhizosphere of bx1 mutants (31). Thus, iron supply and benzoxazinoids likely interact to determine leaf iron homeostasis. In support of this hypothesis, we find that WT plants contain more iron in their leaves than $b x 1$ plants when grown in soils in which iron is available in free or weakly complexed form but not in soil in which iron availability is low (Fig. 1 and SI Appendix, Figs. S4 and S7). To further explore this aspect, we measured the expression of genes involved in iron homeostasis in the leaves of WT and $b x 1$ mutant plants grown under different forms of iron supply (Fig. 3 and SI Appendix, Fig. S8). The tested genes included genes associated with iron transport, such as ZmYS1, $Z m N R A M P 1$, and $Z m I R O 2$, and genes that are likely involved in the biosynthesis and efflux of the mugineic acid family of siderophores, such as ZmRP1, ZmIDI4, ZmNAS3, ZmDMAS1, and ZmTOM2 (35 37). We found strong interactions between iron availability and the $b x 1$ mutation for seven of the eight 

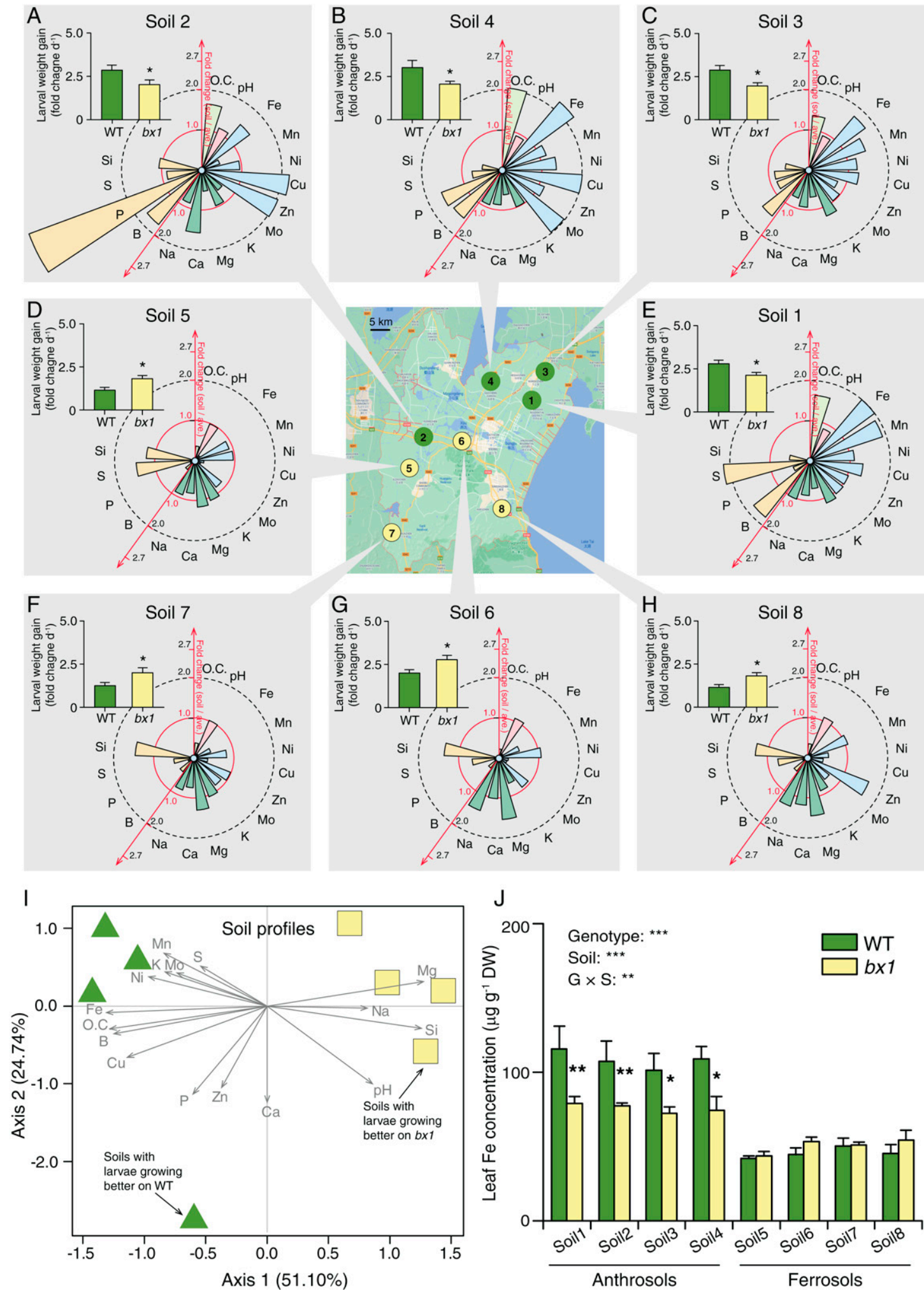

Fig. 1. The effect of benzoxazinoids on herbivore performance depends on the soil type. (Center) Map depicting soil collection sites around Yixing (China). Gray boxes $(A H)$ : Growth of S. frugiperda caterpillars on WT and benzoxazinoid deficient bx1 mutant plants growing in the different soils (+SE, $n=10$ ), together with respective soil properties. Soil properties are depicted as fold change relative to the average across all tested soils. Refer to $S I$ Appendix, Fig. $\mathrm{S} 1$ for absolute values. Soils 1 through 4 are anthrosols, and soils 5 through 8 are ferrosols. Asterisks indicate significant differences between plant genotypes (ANOVA; $* P<0.05$ ). (I) PCA of field soil properties. Green triangles represent soils on which caterpillars grow better on WT plants. Yellow squares represent soils on which caterpillars grow better on $b x 1$ mutant plants. Vectors of soil parameters are shown as gray arrows. $(J)$ Iron contents in the leaves of WT and bx1 plants grown in the different soils ( $+\mathrm{SE}, n=3$, with three to four individual plants pooled per replicate). For full elemental analysis, refer to SI Appendix, Fig. S4. DW, dry weight. O.C., organic carbon. Two way ANOVA results testing for genotype and soil effects are shown $(* * P<0.01 ; * * * P<0.001)$. Asterisks indicate significant differences between genotypes within the same soil (pairwise comparisons through FDR corrected LSMeans; $* P<0.05 ; * * P<0.01$ ). 
A

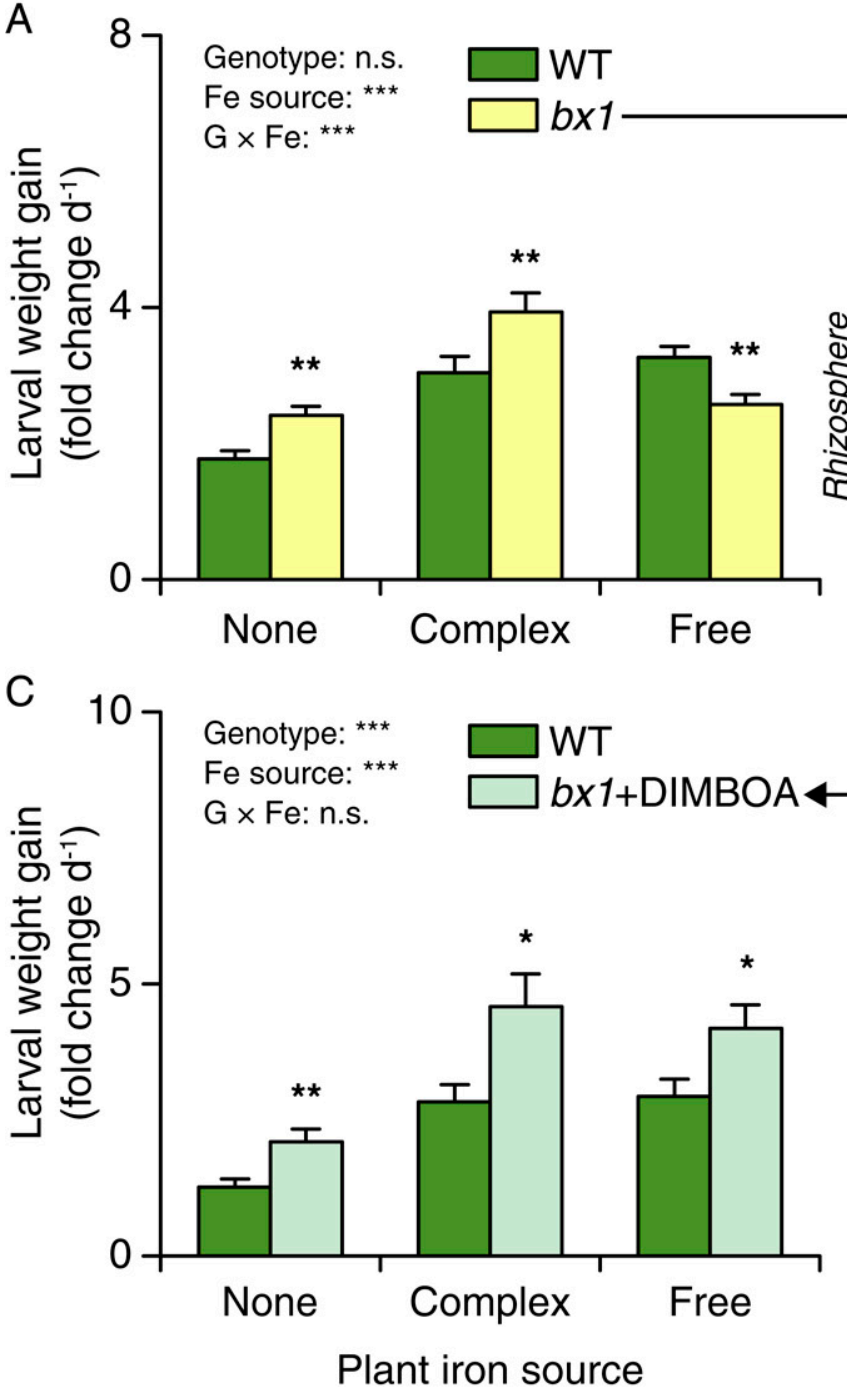

B

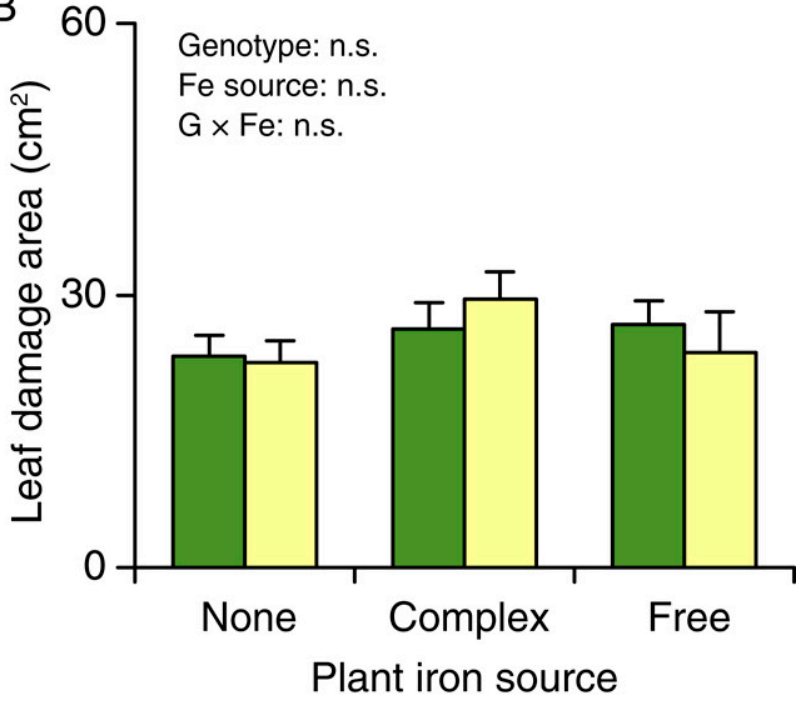

Fig. 2. Soil dependent benzoxazinoid resistance is driven by root iron supply. (A) Growth of S. frugiperda feeding on WT and bx 1 plants supplied with different iron sources ( $+\mathrm{SE}, n=14$ to 15 ). (B) Consumed leaf area ( $+\mathrm{SE}, n=14$ to 15 ). (C) Growth of S. frugiperda feeding on WT and bx1 plants comple mented with pure DIMBOA added to the rhizosphere under different iron sources (+SE, $n=14$ to 15 ). "None" nutrient solutions received either NaCl or $\mathrm{Na}_{2} \mathrm{SO}_{4}$. "Complex" nutrient solutions received Fe EDTA. "Free" nutrient solutions received FeCl $\mathrm{Fr}_{3}$ or $\mathrm{Fe}_{2}\left(\mathrm{SO}_{4}\right)_{3}$. For full results showing genotype effects of all individual nutrient solutions, refer to SI Appendix, Fig. S7. Two way ANOVA results testing for genotype and iron source effects are shown (n.s., not significant; $* * * P<0.001$ ). Asterisks indicate significant differences between genotypes within the same soil (pairwise comparisons through FDR corrected LSMeans; $\left.{ }^{*} P<0.05 ; * * P<0.01\right)$.

tested genes. Most iron homeostasis genes were highly expressed under iron deficient conditions and suppressed in the presence of complex iron, with no differences between WT and $b x 1$ mutant plants. When iron was present in its free form, most of these genes were strongly induced in the $b x 1$ mutant but not in WT plants (Fig. 3). Exceptions to this pattern included $Z m N A S 3$, which showed opposite expression patterns, and $Z m T O M 2$, whose expression was not modulated by the $b x 1$ mutation (Fig. 3). These results show that root iron supply strongly modulates leaf iron homeostasis, with bx1 mutants exhibiting iron deficiency gene expression patterns when grown in the presence of free iron.

Changes in Leaf Herbivore Performance Are Not Explained by Changes in Leaf Primary Metabolism and Defense Expression. How can soil and benzoxazinoid dependent leaf iron homeostasis explain fall armyworm performance? In theory, iron homeosta sis may indirectly affect leaf quality by changing leaf primary metabolism and defense expression (38 40) or directly by act ing as a herbivore micronutrient (23). To test the first hypothe sis, we measured soluble protein, hydrolysable amino acid, and carbohydrate levels in the leaves of WT and $b x 1$ mutant plants grown under different iron regimes (Fig. 4 and SI Appendix, Fig. S9). No significant differences were found, suggesting that the different performance of the fall armyworm is not explained by major changes in leaf primary metabolites.

Next, we measured the production of leaf defense metabo lites that are produced independently of the benzoxazinoid biosynthesis pathway, including chlorogenic acid, rutin, and maysin, which have been associated with fall armyworm resis tance in vitro and through correlative approaches (41 43), and the expression of defense marker genes, including a proteinase inhibitor $(Z m M P I)(44)$ and a gene encoding the defense pro tein RIP2 (ZmRIP2), which is toxic to the fall armyworm in vitro (45). We detected significant interactions between iron availability and the $b x 1$ mutation for rutin and ZmRIP2 

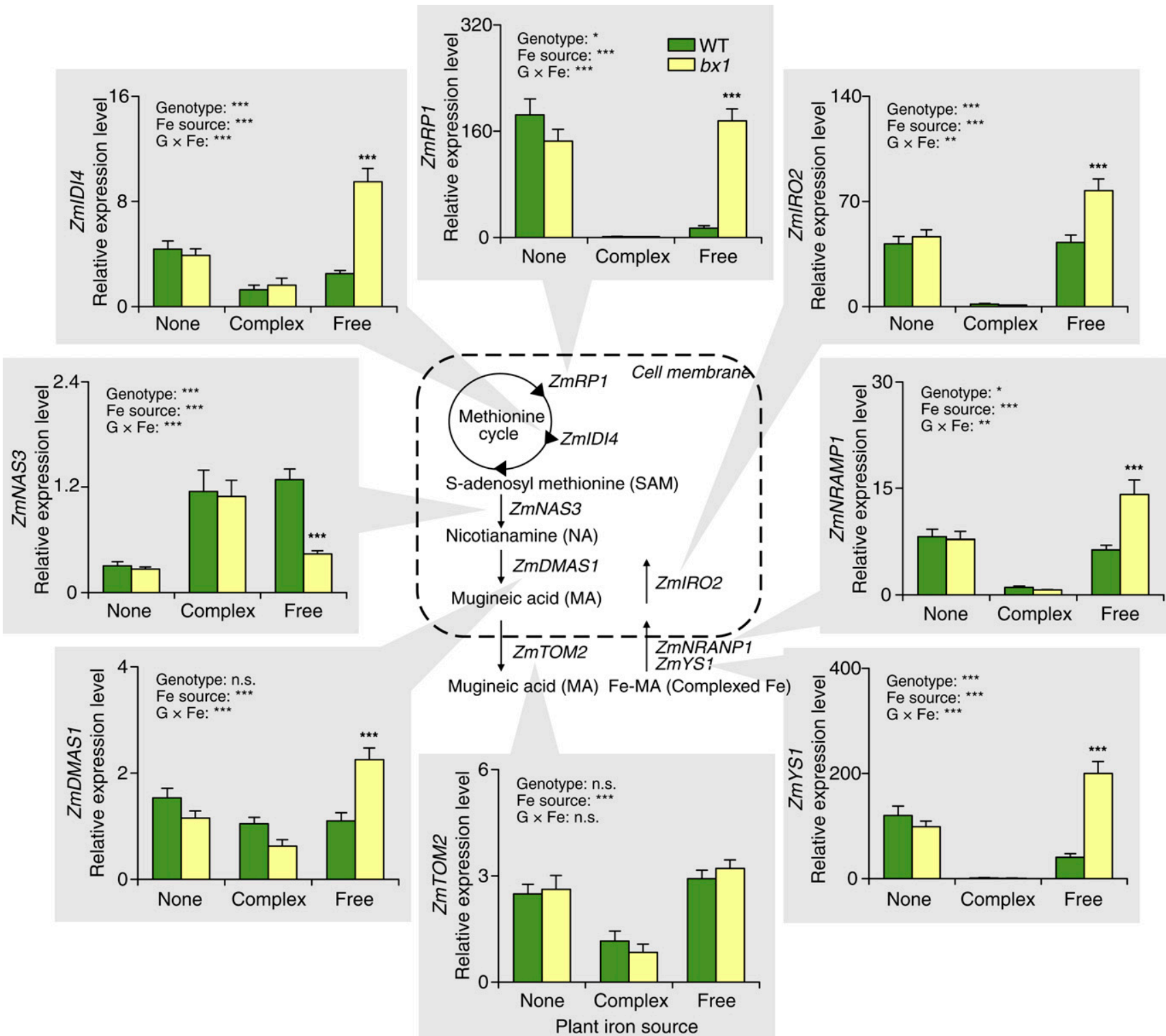

Fig. 3. Interactions between root iron supply and benzoxazinoids determine leaf iron homeostasis. Relative expression of genes involved in iron homeo stasis in the leaves of WT and bx 1 mutant plants supplied with different iron sources ( $+\mathrm{SE}, n=7$ to 8 ). "None" nutrient solutions received either NaCl or $\mathrm{Na}_{2} \mathrm{SO}_{4}$. "Complex" nutrient solutions received Fe EDTA. "Free" nutrient solutions received $\mathrm{FeCl}_{3}$ or $\mathrm{Fe}_{2}\left(\mathrm{SO}_{4}\right)_{3}$. For full results showing genotype effects of all individual nutrient solutions, refer to SI Appendix, Fig. S8. Two way ANOVA results testing for genotype and iron source effects are shown (n.s., not significant; ${ }^{*} P<0.05 ; * * P<0.01 ; * * * P<0.001$ ). Asterisks indicate significant differences between genotypes within the same soil (pairwise comparisons through FDR corrected LSMeans; $* * * P<0.001$ ).

expression (Fig. 5 and SI Appendix, Fig. S10). More rutin was produced in $b x 1$ mutant plants than WT plants grown in the presence of complex iron but not when grown in iron deficient and free iron solutions. ZmRIP2 expression was lower in the $b x 1$ mutant than in WT plants, and these effects were most pronounced when plants were grown in iron deficient and free iron solutions. These results show that root iron supply and benzoxazinoid biosynthesis interact to deter mine leaf defense expression. At the same time, these interac tions are unlikely to explain the observed differences in fall armyworm performance, as patterns do not match. ZmRIP2 expression, for instance, was strongly reduced in the $b x 1$ mutant growing without iron or with iron in its free form, while fall armyworm performance was enhanced on $b x 1$ mutant plants grown without iron but suppressed in $b x 1$ plants grown with free iron.
Herbivore Iron Supply Is Associated with Soil-Dependent Benzoxazinoid Resistance. To test the hypothesis that benzoxazinoids may improve fall armyworm performance by supplying die tary iron, we first screened micronutrient concentrations in fall armyworm larvae fed on WT and bxl mutant plants grown in the different field soils. We found higher levels of iron in larvae feeding on WT than $b x 1$ mutant plants in soil with high iron availability. In soils with low iron availability, larval iron levels were low and not different between plant genotypes (Fig. 6A). No significant effects were found for the other tested elements (SI Appendix, Fig. S11). The same pat tern for iron was observed for $b x 1$ and $b x 2$ mutants in the W22 genetic background (SI Appendix, Fig. S12). Larvae feeding on plants growing in different iron solutions showed corresponding patterns, with significantly lower larval iron levels when feeding on $b x 1$ than WT plants grown together 

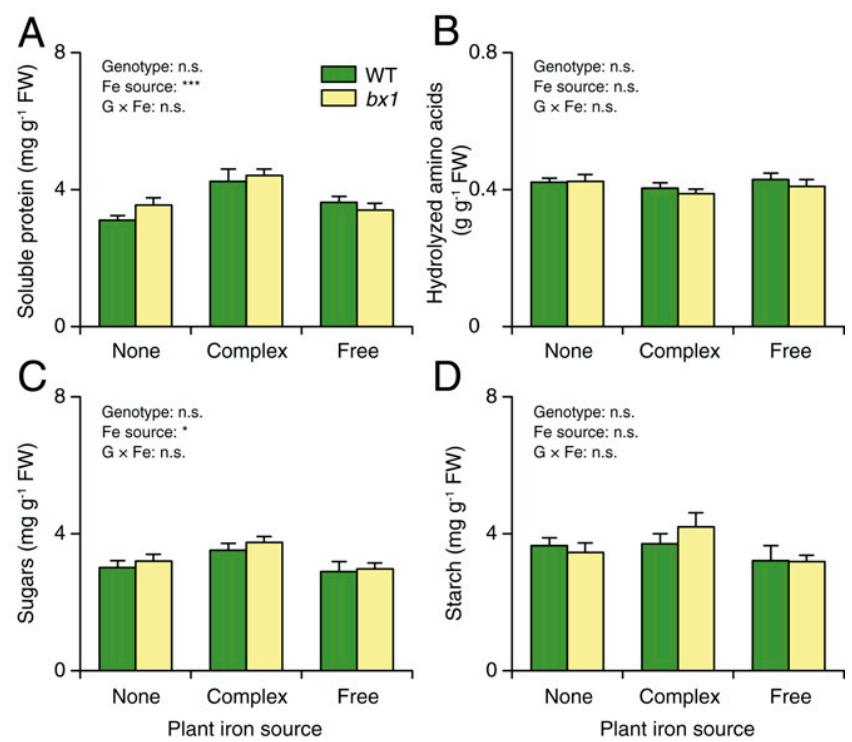

Fig. 4. Changes in leaf herbivore performance are not explained by changes in leaf primary metabolism. Contents of soluble protein $(A)$, hydrolysable amino acids $(B)$, sugars $(C)$, and starch $(D)$ in the leaves of WT and $b \times 1$ mutant plants supplied with different iron sources $(+\mathrm{SE}, n=14$ to 15). "None" nutrient solutions received either $\mathrm{NaCl}$ or $\mathrm{Na}_{2} \mathrm{SO}_{4}$. "Complex" nutrient solutions received Fe EDTA. "Free" nutrient solutions received $\mathrm{FeCl}_{3}$ or $\mathrm{Fe}_{2}\left(\mathrm{SO}_{4}\right)_{3}$. For full results showing genotype effects of all individ ual nutrient solutions, refer to SI Appendix, Fig. S9. Two way ANOVA results testing for genotype and iron source effects are shown (n.s., not significant; $\left.{ }^{*} P<0.05 ; * \star \star P<0.001\right)$. No significant differences between genotypes within the same soil were observed (pairwise comparisons through FDR corrected LSMeans).

with free iron (Fig. $6 B$ and SI Appendix, Fig. S13). Adding DIMBOA to the rhizosphere of bxl mutant plants restored larval iron levels (Fig. 6C), thus providing a direct link between benzoxazinoids in the rhizosphere and larval iron homeostasis. To test whether fall armyworm performance is affected by iron supply, we measured larval growth in the iron transport deficient ys 1 mutant $(35,46)$. Larvae gained less weight in the ys 1 mutant compared to B73 plants (Fig. $6 D)$. As the genetic background of the $y s 1$ is not controlled, other genetic differences may also have influenced fall army worm growth. Thus, we conducted iron supplementation experiments by producing an iron deficient diet and supple menting it with different forms of iron, including the DIMBOA iron complex Fe(III)(DIMBOA) $)_{3}$ at physiological concentrations. Fall armyworm larvae gained more weight when fed on iron supplemented diets, irrespective of the iron source (Fig. 6E). At the tested concentration, DIMBOA alone had no negative effect on fall armyworm growth, which is in accordance with earlier results (32). From these experi ments, we conclude that the interaction between soil micro nutrient composition and benzoxazinoid biosynthesis directly influences iron homeostasis of a leaf herbivore and that these effects can explain the soil dependent impact of benzoxazi noids on herbivore performance.

\section{Discussion}

Organismal traits are commonly coopted for multiple functions, which can lead to important context dependent performance patterns $(5,47)$. Here, we demonstrate that the multifunctional ity of plant secondary metabolites results in conditional out comes of plant herbivore interactions, with soil properties determining whether the secondary metabolites promote or suppress leaf herbivore growth. We discuss the mechanisms and agroecological implications of this finding.

Multifunctionality is a common property of plant secondary metabolites $(16,48,49)$, with potentially important consequen ces for organismal interactions. We find that the protective effect of benzoxazinoids against an herbivore is determined and fully reversible by specific soil properties. A series of manipula tive experiments in combination with the current state of knowledge $(48,50)$ allows us to infer the following scenario. When ingested by herbivores, such as the fall armyworm, ben zoxazinoids are rapidly deglycosylated (32). While the more stable forms, such as DIMBOA, can be detoxified through ster eoselective reglycosylation (51), less stable aglucones, such as HDMBOA, can form reactive hemiacetals that form covalent bonds with thiol groups and can thus act as digestibility reduc ers as well as behavioral modulators $(32,52)$. These effects likely contribute to reduced herbivore weight gain of the fall armyworm in the presence of benzoxazinoids. At the same time, however, benzoxazinoids are also released into the rhizo sphere, where they interact with soil microbes (53) and effec tively chelate free (31) and weakly bound iron, thus making it available to the plant. The higher iron uptake increases leaf iron levels, which benefits herbivores whose growth is limited by iron supply. The net impact of benzoxazinoid biosynthesis on the interaction between maize and leaf herbivores is thus likely governed by the strength of the negative effects of ben zoxazinoids as digestibility reducers and the strength of the pos itive effects of benzoxazinoids as siderophores. By consequence, soil chemistry can tip the balance and determine whether ben zoxazinoids have a net positive or negative effect on herbivore performance.

Plant nutrients in general $(19,22,5457)$ and soil iron supply in particular $(20,21,58)$, are increasingly recognized as impor tant modulators of plant defense expression. In Arabidopsis thaliana, for instance, the coumarin scopoletin is secreted under iron deficiency and influences root microbiome assembly (21), likely including microbes that subsequently trigger sys temic resistance in the plant by activating or priming hormonal defense pathways (20). Another example is $\mathrm{Si}$ in the soil, which can modulate plant nitrogen supply (59) and can also be used directly by plants to form defensive crystals on leaf surfaces (60), thus resulting in significant interactive effects of soil nitro gen and silicon on herbivore performance (61). In our work, we find that benzoxazinoid biosynthesis interacts with root iron supply to regulate a subset of leaf defenses, including the phe nolic acid rutin and messenger RNA levels of the defense pro tein ZmRIP2. These effects are unlikely to be caused by changes in primary metabolism via leaf iron supply, as leaf carbohydrates and amino acids do not show any differences between treatments at this growth stage. Interestingly, patterns of defense expression and leaf iron homeostasis markers also do not correspond: While iron homeostasis is most strongly affected by benzoxazinoids in the presence of free iron, defense expression is most strongly affected by the presence of the iron complex Fe EDTA in the growth medium. Benzoxazinoids shape the rhizosphere microbiome $(53,62,63)$, and this effect may be modulated by competition for iron. It is thus possible that the type of iron source that is present in the growth solu tion has an impact on benzoxazinoid microbiome interactions, which, again, may affect the activation of leaf defenses through systemic signaling. Further experiments will be required to explore this hypothesis. Although the observed modulation of the measured leaf defense metabolites and defense genes may have contributed to fall armyworm growth, they are not directly responsible for the observed benzoxazinoid dependent pat terns, as benzoxazinoids affect fall armyworm growth differ ently in no iron and free iron treatments, without any change in benzoxazinoid mediated defense expression. It should be 

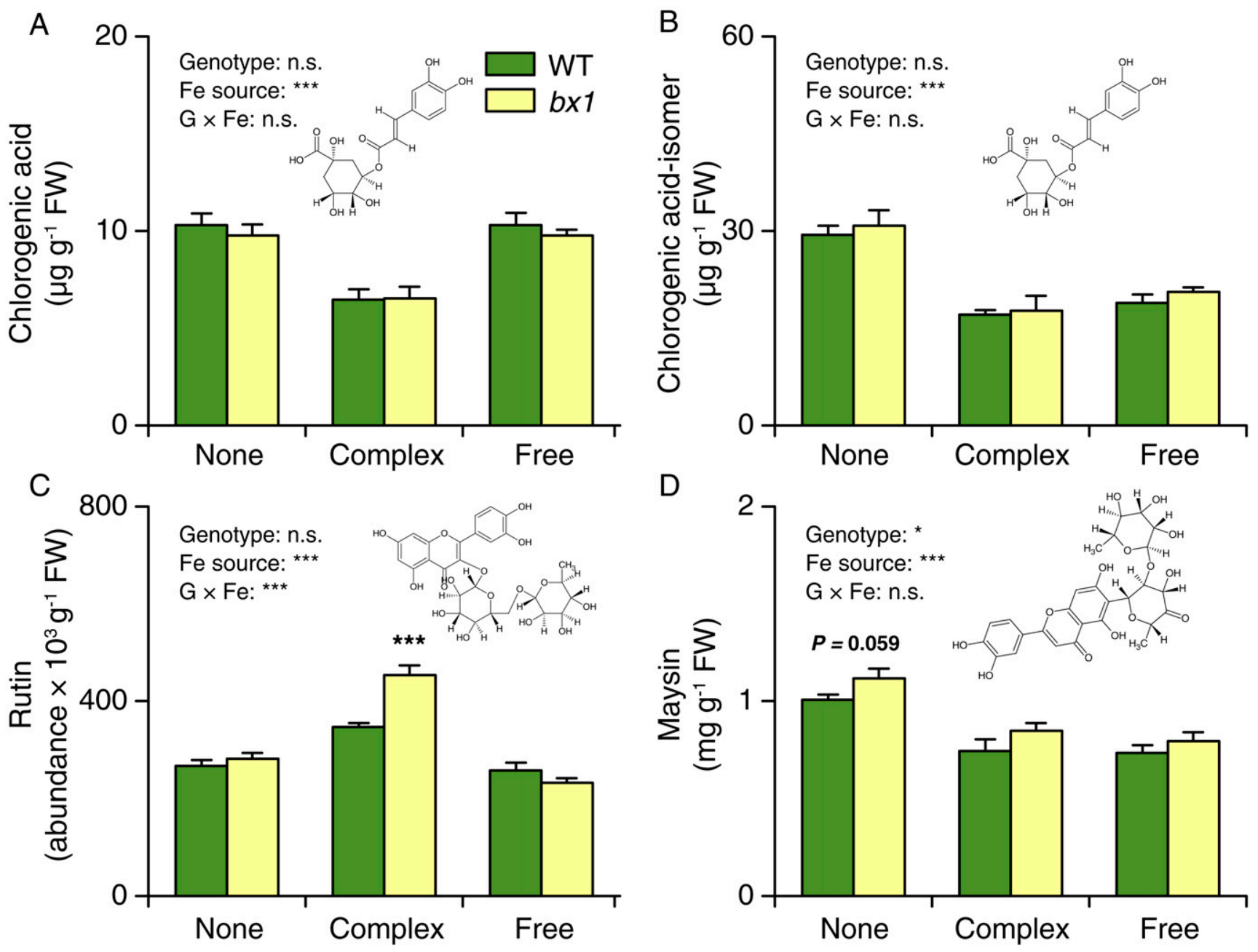

\section{Defense-related genes}
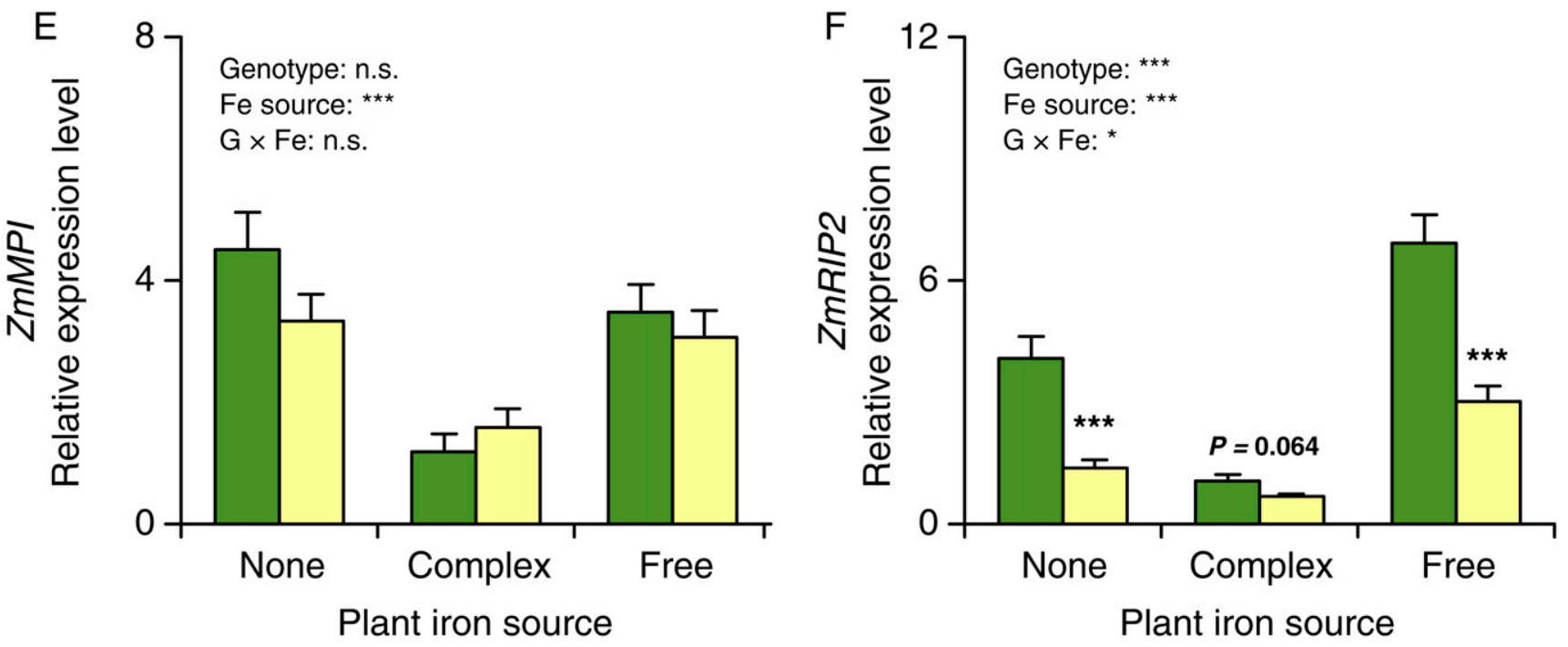

Fig. 5. Soil iron and benzoxazinoids interactively reprogram a subset of leaf defenses. $(A D)$ Concentrations of chlorogenic acid $(A)$, chlorogenic acid iso mer $(B)$, rutin $(C)$, and maysin $(D)$ in the leaves of WT and $b x 1$ mutant plants supplied with different iron sources $(+S E, n=8)$. FW, fresh weight. $(E$ and $F)$ Expression levels of ZmMPI $(E)$ and $Z m R I P 2(F)$ in the leaves of WT and bx1 plants supplied with different iron sources (+SE, $n=8$ ). "None" nutrient solu tions received either $\mathrm{NaCl}$ or $\mathrm{Na}_{2} \mathrm{SO}_{4}$. "Complex" nutrient solutions received Fe EDTA. "Free" nutrient solutions received FeCl 3 or Fe ${ }_{2}\left(\mathrm{SO}_{4}\right)_{3}$. For full results showing genotype effects of all individual nutrient solutions, refer to SI Appendix, Fig. S10. Two way ANOVA results testing for genotype and iron source effects are shown $(* P<0.05 ; * * * P<0.001)$. Asterisks indicate significant differences between genotypes within the same soil (pairwise comparisons through FDR corrected LSMeans; $* * * P<0.001$ ). 


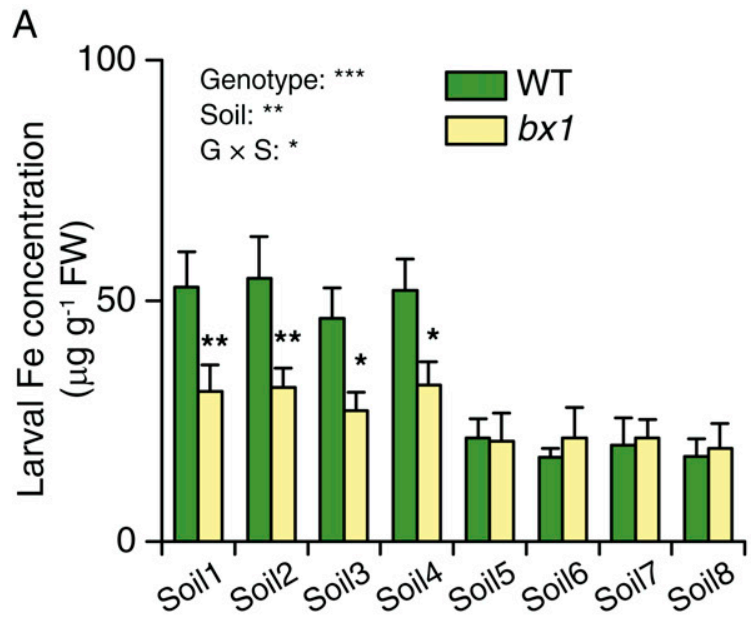

B
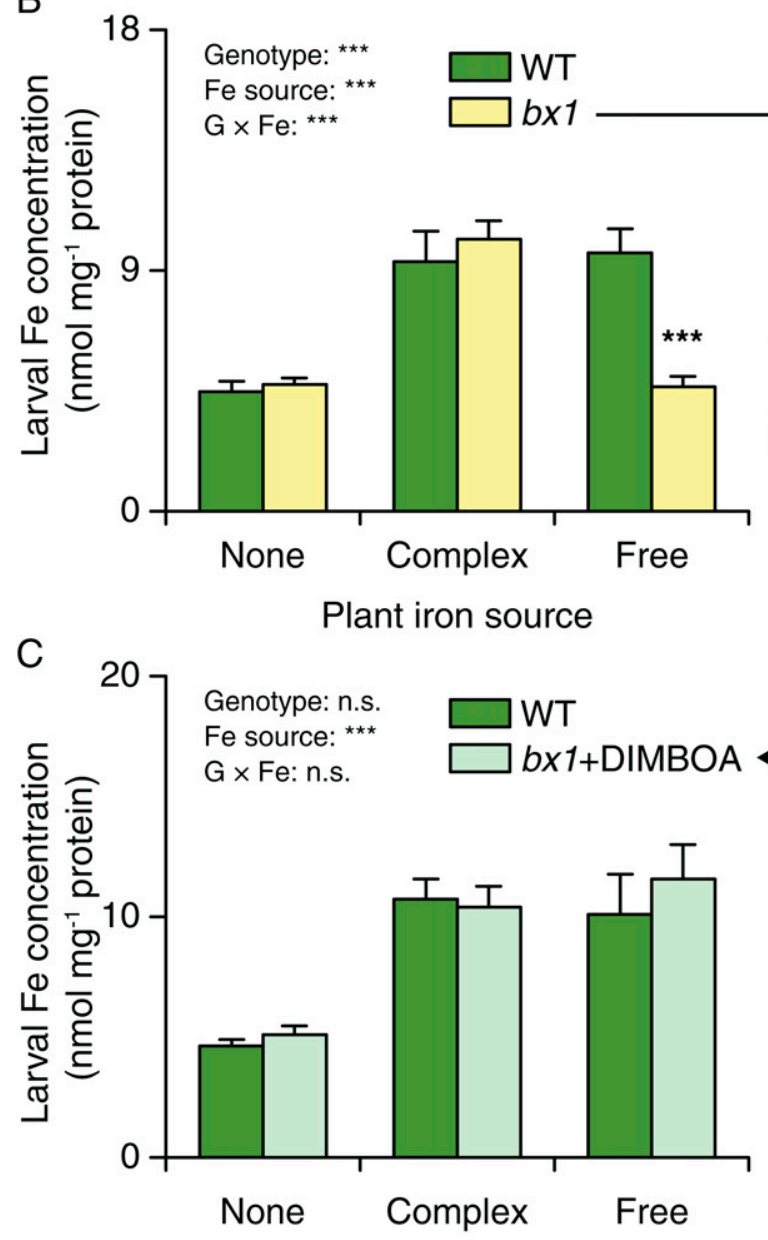

Plant iron source
D

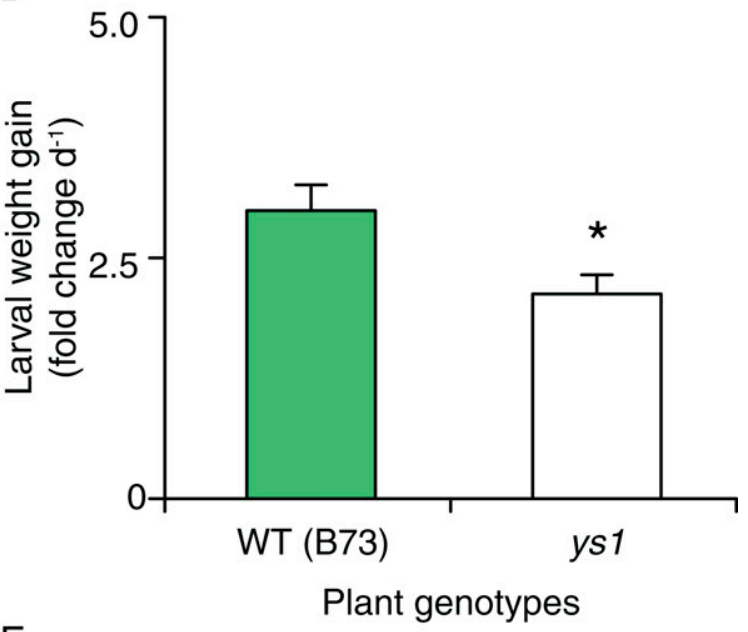

$\mathrm{E}$

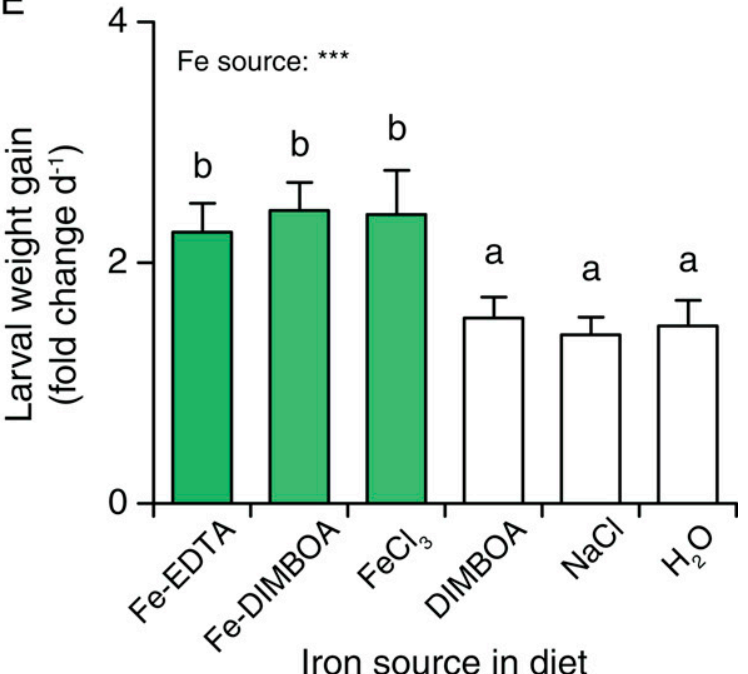

Fig. 6. Herbivore iron supply is associated with soil dependent benzoxazinoid resistance. $(A)$ Iron contents of $S$. frugiperda larvae feeding on WT and bx 1 plants grown in field soils ( $\mathrm{SE}, n=3$, with three to four individual larvae pooled per replicate). Soils 1 through 4 are anthrosols, and soils 5 through 8 are fer rosols. Twoway ANOVA results testing for genotype and soil effects are shown $(* P<0.05 ; * \star P<0.01 ; * * * P<0.001)$. Asterisks indicate significant differences between genotypes within the same soil (pairwise comparisons through FDR corrected LSMeans; ${ }^{\star} P<0.05$; $* * P<0.01$ ). (B and $C$ ) Iron contents of $S$. frugiperda larvae feeding on WT and $b x 1$ plants $(B)$, with DIMBOA rhizosphere complementation $(C)$ under different iron source treatments $(+\mathrm{SE}, n=5$, with three indi vidual larvae pooled per replicate). "None" nutrient solutions received either $\mathrm{NaCl}$ or $\mathrm{Na}_{2} \mathrm{SO}_{4}$. "Complex" nutrient solutions received Fe EDTA. "Free" nutrient solutions received $\mathrm{FeCl}_{3}$ or $\mathrm{Fe}_{2}\left(\mathrm{SO}_{4}\right)_{3}$. For full results showing genotype effects of all individual nutrient solutions, refer to SI Appendix, Fig. S13. Two way ANOVA results testing for genotype and iron source effects are shown ( $* * * P<0.001)$. Asterisks indicate significant differences between genotypes within the same soil (pairwise comparisons through FDR corrected LSMeans; $* * * P<0.001$ ). (D) Growth of $S$. frugiperda larvae feeding on B73 and ys1 mutants (+SE, $n=$ 13 to 16$)$. Note that the ys 1 mutant is in an undefined background. Asterisks indicate significant differences between plant genotypes (ANOVA; $P<0.05)$. (E) Growth of $S$. frugiperda larvae feeding on the artificial diets supplemented with different iron sources (+SE, $n=12$ ). Asterisks and letters indicate significant differences between genotypes or iron sources (one way ANOVA followed by pairwise comparisons through FDR corrected LSMeans; $P<0.05 ; * * *<0.001$ ). 
noted that many other resistance factors apart from the ones measured here could contribute to the observed patterns. Nev ertheless, the currently available data suggests that the direct effects of iron uptake on herbivore performance override potential indirect effects via root microbial communities or iron dependent defense regulation.

Plant herbivore interactions play an important role in shaping ecological communities and agricultural productivity $(64,65)$. Understanding the role of plant secondary metabolites as resis tance factors is thus important for both fields. Our work shows that the suppressive effect of benzoxazinoids on fall armyworm growth, which likely contributes to plant resistance, depends on, and can even be reversed by, soil characteristics. In general, soil and plant chemistry interact to determine the outcome of plant herbivore interactions above ground $(22,54)$. Through sec ondary metabolite multifunctionality, soil properties may deter mine plant and herbivore community composition even more strongly and dynamically than hitherto anticipated (66). From an agricultural point of view, the uncovered dependencies limit the use of benzoxazinoids as natural defenses against the fall army worm, a global invasive pest that is currently threatening maize production in Africa and Asia. The finding that benzoxazinoids do not suppress the growth of the fall armyworm when growing in certain soils is of particular importance in the context of the rapidly expanding maize production in Asia, as it shows the limits of using a core innate defense mechanism to broadly protect agroecosystems from an important invasive pest.

\section{Materials and Methods}

Plants and Insects. The maize (Zea mays L.) genotypes B73 (referred to as WT), bx1/B73 (referred to as bx1) (33), W22, bx1NW22 (bx1::Ds), and bx2N22 (bx2::Ds) (67) were used in this study. ys1 mutants in an undefined genetic background $(36,46)$ were used to test the impacts of plant iron supply on larval performance. Fall armyworm Spodoptera frugiperda (J.E. Smith, 1791) larvae were reared on an artificial diet as described previously (68).

Plant and Herbivore Performance in Field Soils. To determine the impact of available soil nutrients on plant performance and herbivore resistance, we col lected eight soils from different arable fields in Yixing, Jiangsu province, China (SI Appendix, Table S1). The soils were first air dried and then individually passed through a $2 \mathrm{~mm}$ sieve, homogenized, and used to fill $200 \mathrm{~mL}$ pots (11 cm depth and $5 \mathrm{~cm}$ diameter). B73, bx1/B73, W22, bx1/W22, and bx2/N22 plants were then individually grown in these soils. Pots were randomly placed on a greenhouse table $\left(26^{\circ} \mathrm{C} \pm 2{ }^{\circ} \mathrm{C}, 55 \%\right.$ relative humidity, $14: 10 \mathrm{~h}$ light/dark, $50,000 \mathrm{~lm} \cdot \mathrm{m}^{2}$ ) and rearranged weekly. Plants were watered once per week. A total of $16 \mathrm{~d}$ after planting, we measured the shoot dry weight, elemental composition ( $n=3$, with 3 to 4 plants pooled per replicate), and larval growth $(n=10)$ on each maize plant. Chlorophyll contents were determined using an SPAD 502 meter (Minolta Camera Co.).

Plant and Herbivore Performance in Nutrient Solutions. To assess the impact of different forms of iron on plant performance, a soil free growth system was used as described previously (31). Briefly, B73 and $b x 1$ seeds were individ ually wrapped in two layers of paper. The paper rolls with the seeds were put in $200 \mathrm{~mL}$ pots $(11 \mathrm{~cm}$ depth and $5 \mathrm{~cm}$ diameter). Pots were supplied with $40 \mathrm{~mL}$ Milli Q water, covered with aluminum (Al) foil, and then placed in the greenhouse $\left(26^{\circ} \mathrm{C} \pm 2{ }^{\circ} \mathrm{C}, 55 \%\right.$ relative humidity, $14: 10 \mathrm{~h}$ light/dark, $50,000 \mathrm{Im}$ $\cdot \mathrm{m}^{2}$ ). A period of $1 \mathrm{wk}$ after the start of germination, the remaining seed shell was removed from the germinating seedlings to reduce the influence of residual iron in the endosperm. Plants were then grown in nutrient solutions containing complexed iron (Fe EDTA), free iron $\left[\mathrm{FeCl}_{3}, \mathrm{Fe}_{2}\left(\mathrm{SO}_{4}\right)_{3}\right]$, or no iron $\left(\mathrm{NaCl}, \mathrm{Na}_{2} \mathrm{SO}_{4}\right)$ sources. For a complete description of the nutrient solution, see ref. 31. The final concentrations of the different forms of iron in the solu tion were $250 \mu \mathrm{M}$ Fe EDTA, $250 \mu \mathrm{M} \mathrm{FeCl}_{3}$, or $125 \mu \mathrm{M} \mathrm{Fe}_{2}\left(\mathrm{SO}_{4}\right)_{3}$. The respective Fe free control solutions contained $750 \mu \mathrm{M} \mathrm{NaCl}$ or $375 \mu \mathrm{M} \mathrm{Na}_{2} \mathrm{SO}_{4}$ to control for effects of $\mathrm{Cl}, \mathrm{SO}_{4}{ }^{2}$, and $\mathrm{Na}^{+}$in the iron salt treatments. The $\mathrm{pH}$ of the nutrient solutions was adjusted to 5.5 using potassium hydroxide. All the chemicals were bought from Sigma (Sigma Aldrich). A period of $3 \mathrm{wk}$ after germination, we quantified the gene expression, primary and secondary metabolites of the youngest fully developed leaf $(n=8)$, and larval growth on each plant ( $n=14$ to 15$)$.
To determine whether DIMBOA is sufficient to restore the resistance of $b x 1$ plants to those of WT plants, WT and bx 1 plants were treated as described in the previous paragraph. A period of $1 \mathrm{wk}$ after germination, the plants were supplied with nutrient solutions containing Fe EDTA, $\mathrm{FeCl}_{3}$, or $\mathrm{NaCl}$. The nutrient solution for the bx1 mutants was complemented with $300 \mu \mathrm{g}$ DIM BOA, which corresponds to physiological concentrations of DIMBOA that accumulate in the rhizosphere of B73 plants (31). The larval growth on each plant was then assessed as described in Herbivore Growth and Damage Assays $(n=15)$.

To investigate the connections between plant Fe acquisition and larval growth, B73 and ys 1 plants were treated as described above ( $n=13$ to 16$)$. A period of $1 \mathrm{wk}$ after germination, the plants were grown in nutrient solutions supplied with Fe EDTA. A period of 3 wk after germination, larval growth on each plant was recorded.

Soil, Plant, and Herbivore Nutrient Analyses. To characterize nutrients in bulk field soil, soil samples were air dried and then individually ground and passed through a $1 \mathrm{~mm}$ sieve. The available $\mathrm{Fe}, \mathrm{Mn}, \mathrm{Ni}, \mathrm{Cu}$, and $\mathrm{Zn}$ were extracted according to China Environmental Protection standards (HJ 804 2016). Briefly, $10.0 \mathrm{~g}$ soil sample was mixed with $20 \mathrm{~mL}$ extraction buffer $(0.005 \mathrm{M}$ diethyle netriaminepentaacetic acid [DTPA], $0.01 \mathrm{M} \mathrm{CaCl}_{2}, 0.1 \mathrm{M}$ triethanolamine, $\mathrm{pH}=$ 7.3) and then shaken $(180 \mathrm{rpm})$ for $2 \mathrm{~h}$ at $20^{\circ} \mathrm{C}$. DTPA is a strong chelator and has high affinity for metal cations. It can chelate and sequester free and weakly bond metal cations but not strongly chelated metal complexes and metal oxides in soil. After shaking, the mixture was centrifuged, superna tant was collected, and the concentrations of $\mathrm{Fe}, \mathrm{Mn}, \mathrm{Ni}, \mathrm{Cu}$, and $\mathrm{Zn}$ were determined by inductively coupled plasma mass spectrometry (ICP MS) (NexION300X, PerkinElmer). The parameters used during the ICP MS measure ments were the following: radio frequency generator power output: 1,600 W; argon flows: plasma, $1.5 \mathrm{~L} \cdot \min { }^{1}$; nebulizer: $1.09 \mathrm{~L} \cdot \min { }^{1}$, kinetic energy dis crimination gas: helium, at flow $3.5 \mathrm{~mL} \cdot \min { }^{1}$; optimization on masses of ${ }^{9} \mathrm{Be},{ }^{24} \mathrm{Mg},{ }^{115} \mathrm{In},{ }^{238} \mathrm{U},{ }^{140} \mathrm{Ce}$; data acquisition: dwell time of $50 \mathrm{~ms}$, three points per peak, acquisition time of $3 \mathrm{~s} .{ }^{57} \mathrm{Fe},{ }^{55} \mathrm{Mn},{ }^{60} \mathrm{Ni},{ }^{63} \mathrm{Cu}$, and ${ }^{66} \mathrm{Zn}$ were used as analytical masses to reduce interferences. A $40 \mu \mathrm{g} \cdot \mathrm{L}{ }^{1} \mathrm{Rh}$ solution as an inter nal standard in order to compensate any possible signal instability and a wash ing cycle of at least $30 \mathrm{~s}$ were settled between two subsequent samples with the aim to eliminate any memory effects. All reported data were blank cor rected. In order to monitor constantly the overall accuracy level of the method, a blank was run up every eight samples. A standard reference mate rial, certified reference material Cabbage, GBW10014 (GSB 5), prepared by the Institute of Geophysical and Geochemical Exploration of China, was run every 12 samples to determine the accuracy of the analytical methods. The mixed standard samples (PerkinElmer, catalog No. N9300233) from $1 \mu \mathrm{g} \cdot \mathrm{L}{ }^{1}$ to $200 \mu \mathrm{g} \cdot \mathrm{L}{ }^{1}$ were used to build a standard curve, with correlation coeffi cient $\left(R^{2}\right)$ of each element being higher than 0.999 . The absolute quantities of $\mathrm{Fe}, \mathrm{Mn}, \mathrm{Ni}, \mathrm{Cu}$, and $\mathrm{Zn}$ were calculated according to the standard curve.

Soil $\mathrm{pH}, \mathrm{NH}_{4}{ }^{+}$, available $\mathrm{P}, \mathrm{S}, \mathrm{Si}, \mathrm{B}, \mathrm{Mo}$, and exchangeable $\mathrm{K}^{+}, \mathrm{Na}^{+}, \mathrm{Ca}^{2+}$, and $\mathrm{Mg}^{2+}$ were extracted and determined according to the China National Standard Methods. Briefly, soil pH was determined in a 2.5:1 water/soil suspension using a pH meter (LY/T1239 1999). $\mathrm{NH}_{4}{ }^{+}$was measured using alkali hydrolysis diffusion (LY/T 1231 1999). Available $\mathrm{P}$ was determined by hydro chloric acid and ammonium fluoride (LY/T 1233 1999). Available $S$ was extracted with calcium phosphate acetic acid and quantified with the turbidi metric method using barium sulfate (LY/T 1265 1999). Available Si was extracted by sodium acetate and quantified by the silicon molybdenum blue colorimetry (LY/T 1266 1999). Available B was extracted by boiled deionized water and determined by the azomethine $\mathrm{H}$ spectrophotometric method (LY/ T 1258 1999). Available Mo was extracted by acid ammonium oxalate and quantified by colorimetry using potassium thiocyanate (LY/T 1259 1999). Exchangeable $\mathrm{K}^{+}, \mathrm{Na}^{+}, \mathrm{Ca}^{2+}$, and $\mathrm{Mg}^{2+}$ were extracted by ammonium acetate and determined by flame photometry (LY/T 1246 1999) and atomic absorption spectrophotometry (LY/T 1245 1999), respectively.

For plant micronutrient analyses, plant leaves were oven dried. Three or four individual plants were pooled as one replicate. The samples were digested in $6 \mathrm{~mL} 15 \mathrm{M} \mathrm{HNO}_{3}$ and $10 \mathrm{M} \mathrm{H}_{2} \mathrm{O}_{2}$ at $190^{\circ} \mathrm{C}$ for $35 \mathrm{~min}$ with MARS 6 CLASSIC (CEM Corp.) as described (69). After digestion, the samples were dis solved in deionized water. The concentrations of $\mathrm{Mg}, \mathrm{Fe}, \mathrm{Mn}, \mathrm{Ni}, \mathrm{Cu}$, and $\mathrm{Zn}$ were determined by ICP MS as described in the previous paragraph. The con centrations of $\mathrm{K}, \mathrm{Ca}, \mathrm{Na}, \mathrm{P}, \mathrm{Si}, \mathrm{B}$, and Mo were quantified by the ICP optical emission spectrometric method according to the China National Standard Method (GB/T 35871 2018).

For micronutrient analyses of fall armyworm larvae, three or four larvae were pooled together as one replicate. The elements were extracted and determined as described. 
Herbivore Growth and Damage Assays. To assess the S. frugiperda growth on maize plants, individual starved and preweighted second instar larvae were introduced into cylindrical mesh cages $(1 \mathrm{~cm}$ height and $2.5 \mathrm{~cm}$ diameter). The cages were then clipped onto the leaves of maize plants (one cage per plant). The position of each cage was moved every day to provide sufficient food sup ply for the larvae. Larval weight was recorded $5 \mathrm{~d}$ after the start of the experi ment. The remaining leaves were scanned, and the removed leaf area was quantified using Digimizer 4.6.1 (Digimizer).

Primary Metabolite Analyses. Soluble protein was extracted and quantified using a Bradford assay $(n=8)(70)$. Amino acids were hydrolyzed and quanti fied by ultra performance liquid chromatography mass spectrometer (UHPLC MS, Waters Corporation) according to a previously published protocol $(n=8)$ (71). Starch and soluble sugars (glucose, sucrose, and fructose) were extracted and quantified as described previously $(n=8)(72)$.

Secondary Metabolite Analyses. To quantify the influences of Fe forms on sec ondary metabolites, three maize plants were pooled, homogenized, and ground by liquid nitrogen ( $n=4$ pools per Fe treatment). A total of $70 \mathrm{mg}$ ground samples was extracted in $700 \mu \mathrm{L}$ acidified $\mathrm{H}_{2} \mathrm{O} / \mathrm{MeOH}(50: 50 \mathrm{vol} / \mathrm{vol}$; $0.1 \%$ formic acid) and then analyzed with an Acquity UHPLC system coupled to a G2 XS quadrupole time of flight (QTOF) MS equipped with an electro spray source (Waters Corporation) as described (53). Briefly, compounds were separated on an Acquity BEH C18 column $(2.1 \times 50 \mathrm{~mm}$ inner diameter, 1.7 $\mu \mathrm{m}$ particle size). Water ( $0.1 \%$ formic acid) and acetonitrile ( $0.1 \%$ formic acid) were employed as mobile phases $A$ and $B$. The elution profile was the follow ing: 0 to $3.50 \mathrm{~min}, 99$ to $72.5 \% \mathrm{~A}$ in $\mathrm{B} ; 3.50$ to $5.50 \mathrm{~min}, 72.5$ to $50 \% \mathrm{~B} ; 5.51$ to $6.50 \mathrm{~min} 100 \% \mathrm{~B} ; 6.51$ to $7.51 \mathrm{~min}, 99 \% \mathrm{~A}$ in $B$. The flow rate was $0.4 \mathrm{~mL} / \mathrm{min}$. The column temperature was maintained at $40^{\circ} \mathrm{C}$, and the injection volume was $1 \mu \mathrm{L}$. The QTOF MS was operated in negative mode. The data were acquired over an $\mathrm{m} / \mathrm{z}$ range of 50 to 1,200 with scans of $0.15 \mathrm{~s}$ at collision energy of $4 \mathrm{~V}$ and $0.2 \mathrm{~s}$ with a collision energy ramp from 10 to $40 \mathrm{~V}$. The capil lary and cone voltages were set to $2 \mathrm{kV}$ and $20 \mathrm{~V}$, respectively. The source tem perature was maintained at $140^{\circ} \mathrm{C}$, the desolvation was $400^{\circ} \mathrm{C}$ at $1,000 \mathrm{~L} \cdot \mathrm{h}{ }^{1}$, and cone gas flows were $50 \mathrm{~L} / \mathrm{h}$. Accurate mass measurements $(<2 \mathrm{ppm})$ were obtained by infusing a solution of leucin encephalin at $200 \mathrm{ng} / \mathrm{mL}$ at a flow rate of $10 \mathrm{~mL} / \mathrm{min}$ through the Lock Spray probe (Waters Corporation). The relative abundance of rutin was determined based on peak areas. The abso lute quantities of chlorogenic acid, chlorogenic acid isomer, and maysin were determined using standard curves obtained from synthetic or puri fied standards.

Gene Expression Analyses. qRT PCR was used to quantify gene expressions. Total RNA was isolated from maize leaves using the GeneJET Plant RNA Purifi cation Kit (Thermo Fisher Scientific) following the manufacturer's instructions $(n=8)$. A total of $300 \mathrm{ng}$ of each total RNA sample was reverse transcribed with SuperScript II Reverse Transcriptase (Invitrogen). The qRT PCR assay was performed on the LightCycler 96 Instrument (Roche) using the KAPA SYBR FAST qPCR Master Mix (Kapa Biosystems). The maize actin gene ZmActin was used as an internal standard to normalize complementary DNA concentra tions. The relative gene expression levels of target genes were calculated using the $2^{\Delta \Delta \mathrm{Ct}}$ method (73). The primers of all tested genes are provided in $\mathrm{SI}$ Appendix, Table $\mathrm{S} 2$.

Larval Iron Analyses. To determine iron concentrations in S. frugiperda larvae, three larvae were pooled and homogenized ( $n=5$ pools). Total protein of the larval lysates was extracted with $200 \mu \mathrm{L}$ lysis buffer $(20 \mathrm{mM}$ Tris, $137 \mathrm{mM} \mathrm{NaCl}$, $1 \%$ Triton X 100, $1 \%$ glycerol). The concentrations of extracted protein were quantified with a Bradford assay and then denatured using a described protocol (74). After denaturation, $50 \mu \mathrm{L}$ supernatant was taken for iron

1. S. C. Farina, E. A. Kane, L. P. Hernandez, Multifunctional structures and multistructural functions: Integration in the evolution of biomechanical systems. Integr. Comp. Biol. 59, 338-345 (2019).

2. D. S. Tawfik, Messy biology and the origins of evolutionary innovations. Nat. Chem. Biol. 6, 692-696 (2010).

3. R. J. Greenspan, The flexible genome. Nat. Rev. Genet. 2, 383-387 (2001).

4. E. H. Neilson, J. Q. D. Goodger, I. E. Woodrow, B. L. Møller, Plant chemical defense: At what cost? Trends Plant Sci. 18, 250-258 (2013).

5. N. R. Friedman et al., Evolution of a multifunctional trait: Shared effects of foraging ecology and thermoregulation on beak morphology, with consequences for song evolution. Proc. Biol. Sci. 286, 20192474 (2019).

6. B. Li et al., Convergent evolution of a metabolic switch between aphid and caterpillar resistance in cereals. Sci. Adv. 4, eaat6797 (2018).

7. R. Li et al., Prioritizing plant defence over growth through WRKY regulation facilitates infestation by non-target herbivores. eLife 4, e04805 (2015). measurements using the Iron Assay Kit (Sigma) following the manufacturer's instructions, with the modification that $25 \mu \mathrm{L}$ saturated ammonium acetate was added and mixed to adjust the $\mathrm{pH}$ before measuring absorbance at 593 $\mathrm{nm}$. The absolute quantities of iron were determined using standard curves made from pure $\mathrm{FeCl}_{3}$ according to the manufacturer's instructions.

Larval Growth on Diets with Exogenous Iron Sources. To evaluate the direct effect of iron on S. frugiperda growth, we prepared the artificial diets contain ing different iron sources according to the methods as described in ref. 75, with some modifications. Briefly, $17 \mathrm{~g}$ agar was dissolved in $500 \mathrm{~mL}$ water at $50^{\circ} \mathrm{C}$ and mixed with $5 \mathrm{~g}$ dried leaf material of bx 1 plants, $25 \mathrm{~g}$ casein, $23 \mathrm{~g}$ sucrose, $12 \mathrm{~g}$ yeast extract, $9 \mathrm{~g}$ Wesson salt mixture, $3.5 \mathrm{~g}$ ascorbic acid, $2.5 \mathrm{~g}$ cholesterol, $1.5 \mathrm{~g}$ sorbic acid, $5 \mathrm{~mL}$ raw linseed oil, $1.5 \mathrm{~mL}$ formalin, and $9 \mathrm{~mL}$ vitamin mixture (100 mg nicotinic acid, $500 \mathrm{mg}$ riboflavin, $233.5 \mathrm{mg}$ thia mine, $233.5 \mathrm{mg}$ pyridoxine, $233.5 \mathrm{mg}$ folic acid, and $20 \mathrm{mg} \cdot \mathrm{L}^{1}$ biotin in water). Fe EDTA, Fe(III)(DIMBOA) $)_{3}, \mathrm{FeCl}_{3}$, DIMBOA, $\mathrm{NaCl}$, or $\mathrm{H}_{2} \mathrm{O}$ was then added to the diet at a final concentration of $50 \mu \mathrm{M}$, which corresponds to the physiological concentration in maize xylem sap (31). The produced diet was aliquoted into Solo cups. One starved and preweighted second instar larva was individually introduced into the Solo cups. Diets were replaced every other day. Larval weight was recorded $5 \mathrm{~d}$ after the start of the experiment $(n=12)$.

Statistical Analyses. Larval growth, leaf damage, gene expression, and metab olite data were analyzed by ANOVA followed by pairwise or multiple compar isons of least squares means (LSMeans), which were corrected using the false discovery rate (FDR) method (76). The tested factors and interactions are pro vided directly in the figures. Normality was verified by inspecting residuals, and homogeneity of variance was tested through the Shapiro Wilk tests using the "plotresid" function of the R package "RVAideMemoire" (77). Datasets that did not fit assumptions (Figs. $2 B$ and $C, 3,5 A, E$, and $F$, and $6 B$ and $E$ and SI Appendix, S4 $C$ and $L, S 8$, and S10 $A, E$, and $F$ ) were natural log transformed. For the redundancy analysis, raw data were first scaled with the "scale" function in R. PCAs were then performed with the "MVA" func tion of the "RVAideMemoire" package and the "rda" function of the "vegan" package $(77,78)$. All statistical analyses were conducted with $\mathrm{R} 3.4 .4$ ( $R$ Foundation for Statistical Computing) using the packages "car," "emmeans," and "RVAideMemoire" (77 80).

Accession Numbers. The sequence data of maize genes can be found in the MaizeGDB database under the following accession numbers ZmActin (GRMZM2G126010), ZmMPI (GRMZM2G028393), ZmRIP2 (GRMZM2G119705), ZmRPI (GRMZM2G035599), ZmIDI4 (GRMZM2G067265), ZmNAS3 (GRMZM 2G478568), ZmDMAS1 (GRMZM2G060952), ZmTOM2 (GRMZM5G877788), ZmYS1 (GRMZM2G156599), ZmNRAMP1 (GRMZM2G178190) and ZmIRO2 (GRMZM2G057413).

Data Availability. All the raw data supporting the findings of this study can be downloaded from the DRYAD repository (doi: 10.5061/dryad.0k6djhb15).

ACKNOWLEDGMENTS. We thank Professor Nicolaus von Wirén from Institut für Pflanzengenetik und Kulturpflanzenforschung Gatersleben for sharing the ys1 mutants and Dr. Xianwen Zhang from Zhejiang University for propa gating the seeds. This work was supported by the National Natural Science Foundation of China (41721001, 42007029, and 42090061), the Young Elite Scientists Sponsorship Program by the China Association for Science and Tech nology (2020QNRC002), the Interfaculty Research Collaboration "One Health" of the University of Bern, the Fundamental Research Funds for the Central Universities (2020QNA6009), and the Schweizerischer Nationalfonds zur Förderung der Wissenschaftlichen Forschung (310030 189071).

8. T. Hartmann, From waste products to ecochemicals: Fifty years research of plant secondary metabolism. Phytochemistry 68, 2831-2846 (2007)

9. A. Mithöfer, W. Boland, Plant defense against herbivores: Chemical aspects. Annu. Rev. Plant Biol. 63, 431-450 (2012).

10. N. K. Clay, A. M. Adio, C. Denoux, G. Jander, F. M. Ausubel, Glucosinolate metabolites required for an Arabidopsis innate immune response. Science 323, 95-101 (2009).

11. G. Agati, M. Tattini, Multiple functional roles of flavonoids in photoprotection. New Phytol. 186, 786-793 (2010).

12. M. Huang et al., The major volatile organic compound emitted from Arabidopsis thaliana flowers, the sesquiterpene (E)- $\beta$-caryophyllene, is a defense against a bacterial pathogen. New Phytol. 193, 997-1008 (2012).

13. N. B. Schmid et al., Feruloyl-CoA 6'-Hydroxylase1-dependent coumarins mediate iron acquisition from alkaline substrates in Arabidopsis. Plant Physiol. 164, 160-172 (2014). 
14. E. Soubeyrand et al., The peroxidative cleavage of kaempferol contributes to the biosynthesis of the benzenoid moiety of ubiquinone in plants. Plant Cell 30, 2910-2921 (2018).

15. M. Erb, D. J. Kliebenstein, Plant secondary metabolites as defenses, regulators, and primary metabolites: The blurred functional trichotomy. Plant Physiol. 184, 39-52 (2020).

16. E. Pichersky, R. A. Raguso, Why do plants produce so many terpenoid compounds? New Phytol. 220, 692-702 (2018).

17. D. J. Ballhorn, A. Pietrowski, R. Lieberei, Direct trade-off between cyanogenesis and resistance to a fungal pathogen in lima bean (Phaseolus lunatus L.). J. Ecol. 98 226-236 (2010)

18. D. Baek, H. J. Chun, D. J. Yun, M. C. Kim, Cross-talk between phosphate starvation and other environmental stress signaling pathways in plants. Mol. Cells 40, 697-705 (2017).

19. L. A. J. Mur, C. Simpson, A. Kumari, A. K. Gupta, K. J. Gupta, Moving nitrogen to the centre of plant defence against pathogens. Ann. Bot. 119, 703-709 (2017)

20. A. Martínez-Medina, S. C. M. Van Wees, C. M. J. Pieterse, Airborne signals from Trichoderma fungi stimulate iron uptake responses in roots resulting in priming of jasmonic acid-dependent defences in shoots of Arabidopsis thaliana and Solanum lycopersicum. Plant Cell Environ. 40, 2691-2705 (2017).

21. I. A. Stringlis et al., MYB72-dependent coumarin exudation shapes root microbiome assembly to promote plant health. Proc. Natl. Acad. Sci. U.S.A. 115, E5213-E5222 (2018).

22. D. Debona, F. A. Rodrigues, L. E. Datnoff, Silicon's role in abiotic and biotic plant stresses. Annu. Rev. Phytopathol. 55, 85-107 (2017).

23. J. D. Schade, M. Kyle, S. E. Hobbie, W. F. Fagan, J. J. Elser, Stoichiometric tracking of soil nutrients by a desert insect herbivore. Ecol. Lett. 6, 96-101 (2003)

24. E. R. D. Moise, J. N. McNeil, S. E. Hartley, H. A. L. Henry, Plant silicon effects on insect feeding dynamics are influenced by plant nitrogen availability. Entomol. Exp. Appl. 167, 91-97 (2019).

25. M. Frey, K. Schullehner, R. Dick, A. Fiesselmann, A. Gierl, Benzoxazinoid biosynthesis, a model for evolution of secondary metabolic pathways in plants. Phytochemistry 70 1645-1651 (2009).

26. H. M. Niemeyer, Hydroxamic acids derived from 2-hydroxy-2H-1,4-benzoxazin-3(4H) one: Key defense chemicals of cereals. J. Agric. Food Chem. 57, 1677-1696 (2009).

27. S. Ahmad et al., Benzoxazinoid metabolites regulate innate immunity against aphids and fungi in maize. Plant Physiol. 157, 317-327 (2011)

28. L. N. Meihls et al., Natural variation in maize aphid resistance is associated with 2,4 dihydroxy-7-methoxy-1,4-benzoxazin-3-one glucoside methyltransferase activity. Plant Cell 25, 2341-2355 (2013).

29. C. A. M. Robert et al., A specialist root herbivore exploits defensive metabolites to locate nutritious tissues. Ecol. Lett. 15, 55-64 (2012)

30. L. Bigler, A. Baumeler, C. Werner, M. Hesse, Detection of noncovalent complexes of hydroxamic-acid derivatives by means of electrospray mass spectrometry. Helv. Chim. Acta 79, 1701-1709 (1996)

31. L. Hu et al., Plant iron acquisition strategy exploited by an insect herbivore. Science 361, 694-697 (2018)

32. G. Glauser et al., Induction and detoxification of maize 1,4-benzoxazin-3-ones by insect herbivores. Plant J. 68, 901-911 (2011).

33. D. Maag et al., Highly localized and persistent induction of $B \times 1$-dependent herbivore resistance factors in maize. Plant J. 88, 976-991 (2016).

34. C. Poschenrieder, R. P. Tolrà, J. Barceló, A role for cyclic hydroxamates in aluminium resistance in maize? J. Inorg. Biochem. 99, 1830-1836 (2005).

35. C. Curie et al., Maize yellow stripe1 encodes a membrane protein directly involved in Fe(III) uptake. Nature 409, 346-349 (2001)

36. S. N. Chorianopoulou, Y. I. Saridis, M. Dimou, P. Katinakis, D. L. Bouranis, Arbuscular mycorrhizal symbiosis alters the expression patterns of three key iron homeostasis genes, ZmNAS1, ZmNAS3, and ZmYS1, in S deprived maize plants. Front Plant Sci 6 257 (2015)

37. T. Nozoye, H. Nakanishi, N. K. Nishizawa, Characterizing the crucial components of iron homeostasis in the maize mutants ys1 and ys3. PLoS One 8, e62567 (2013)

38. M. Jahangir, I. B. Abdel-Farid, Y. H. Choi, R. Verpoorte, Metal ion-inducing metabolite accumulation in Brassica rapa. J. Plant Physiol. 165, 1429-1437 (2008).

39. G. Vigani et al., Knocking down mitochondrial iron transporter (MIT) reprograms primary and secondary metabolism in rice plants. J. Exp. Bot. 67, 1357-1368 (2016).

40. J. H. Herlihy, T. A. Long, J. M. McDowell, Iron homeostasis and plant immune responses: Recent insights and translational implications. J. Biol. Chem. 295, 13444-13457 (2020)

41. B. R. Wiseman, R. C. Gueldner, R. E. Lynch, R. F. Severson, Biochemical activity of centipedegrass against fall armyworm larvae. J. Chem. Ecol. 16, 2677-2690 (1990).

42. T. R. F. B. Silva et al., Effect of the flavonoid rutin on the biology of Spodoptera frugiperda (Lepidoptera: Noctuidae). Acta Sci. Agron. 38, 165-170 (2016).

43. B. R. Wiseman, M. E. Snook, D. J. Isenhour, J. A. Mihm, N. W. Widstrom, Relationship between growth of corn earworm and fall armyworm larvae (Lepidoptera: Noctuidae) and maysin concentration in corn silks. J. Econ. Entomol. 85, 2473-2477 (1992).

44. M. C. Tamayo, M. Rufat, J. M. Bravo, B. San Segundo, Accumulation of a maize pro teinase inhibitor in response to wounding and insect feeding, and characterization of its activity toward digestive proteinases of Spodoptera littoralis larvae. Planta $\mathbf{2 1 1}$ 62-71 (2000)

45. W. P. Chuang et al., Caterpillar attack triggers accumulation of the toxic maize protein RIP2. New Phytol. 201, 928-939 (2014).

46. N. Von Wiren, S. Mori, H. Marschner, V. Romheld, Iron inefficiency in maize mutant ys 1 (Zea mays L cv yellow-stripe) is caused by a defect in uptake of iron phytosiderophores. Plant Physiol. 106, 71-77 (1994)
47. L. Sack, T. N. Buckley, Trait multi-functionality in plant stress response. Integr. Comp. Biol. 60, 98-112 (2020).

48. S. Zhou, A. Richter, G. Jander, Beyond defense: Multiple functions of benzoxazinoids in maize metabolism. Plant Cell Physiol. 59, 1528-1537 (2018).

49. D. J. Kliebenstein, Plant nutrient acquisition entices herbivore. Science 361, 642-643 (2018).

50. F. C. Wouters, B. Blanchette, J. Gershenzon, D. G. Vassão, Plant defense and herbivore counter-defense: Benzoxazinoids and insect herbivores. Phytochem. Rev. 15, 1127-1151 (2016).

51. F. C. Wouters et al., Reglucosylation of the benzoxazinoid DIMBOA with inversion of stereochemical configuration is a detoxification strategy in lepidopteran herbivores. Angew. Chem. Int. Ed. Engl. 53, 11320-11324 (2014).

52. A. Köhler et al., Within-plant distribution of 1,4-benzoxazin-3-ones contributes to herbivore niche differentiation in maize. Plant Cell Environ. 38, 1081-1093 (2015).

53. L. Hu et al., Root exudate metabolites drive plant-soil feedbacks on growth and defense by shaping the rhizosphere microbiota. Nat. Commun. 9, 2738 (2018)

54. Y. Lou, I. T. Baldwin, Nitrogen supply influences herbivore-induced direct and indirect defenses and transcriptional responses in Nicotiana attenuata. Plant Physiol. 135, 496-506 (2004)

55. T. J. Massad, L. A. Dyer, G. Vega C, Costs of defense and a test of the carbon-nutrient balance and growth-differentiation balance hypotheses for two co-occurring classes of plant defense. PLoS One 7, e47554 (2012).

56. W. C. Wetzel, H. M. Kharouba, M. Robinson, M. Holyoak, R. Karban, Variability in plant nutrients reduces insect herbivore performance. Nature 539, 425-427 (2016).

57. C. Cabot et al., A role for zinc in plant defense against pathogens and herbivores. Front Plant Sci 10, 1171 (2019).

58. E. H. Verbon et al., Iron and immunity. Annu. Rev. Phytopathol. 55, 355-375 (2017).

59. S. N. Johnson et al., Silicon-induced root nodulation and synthesis of essential amino acids in a legume is associated with higher herbivore abundance. Funct. Ecol. 31, 1903-1909 (2017).

60. F. P. Massey, S. E. Hartley, Physical defences wear you down: Progressive and irreversible impacts of silica on insect herbivores. J. Anim. Ecol. 78, 281-291 (2009).

61. S. N. Johnson et al., Siliceous and non-nutritious: Nitrogen limitation increases antiherbivore silicon defences in a model grass. J. Ecol. 10.1111/1365-2745.13755. (2021)

62. E. N. Kudjordjie, R. Sapkota, S. K. Steffensen, I. S. Fomsgaard, M. Nicolaisen, Maize synthesized benzoxazinoids affect the host associated microbiome. Microbiome 7, 59 (2019).

63. T. E. A. Cotton et al., Metabolic regulation of the maize rhizobiome by benzoxazinoids. ISME J. 13, 1647-1658 (2019).

64. E. S. Bakker, M. E. Ritchie, H. Olff, D. G. Milchunas, J. M. Knops, Herbivore impact on grassland plant diversity depends on habitat productivity and herbivore size. Ecol. Lett. 9, 780-788 (2006).

65. T. Ohgushi, Eco-evolutionary dynamics of plant-herbivore communities: Incorporating plant phenotypic plasticity. Curr. Opin. Insect Sci. 14, 40-45 (2016).

66. D. A. Wardle et al., Ecological linkages between aboveground and belowground biota. Science 304, 1629-1633 (2004)

67. V. Tzin et al., Dynamic maize responses to aphid feeding are revealed by a time series of transcriptomic and metabolomic assays. Plant Physiol. 169, 1727-1743 (2015).

68. D. Maag et al., 3- $\beta$-D-Glucopyranosyl-6-methoxy-2-benzoxazolinone (MBOA- $N$-GIC) is an insect detoxification product of maize 1,4-benzoxazin-3-ones. Phytochemistry 102, 97-105 (2014)

69. H. Yuan et al., Warming facilitates microbial reduction and release of arsenic in flooded paddy soil and arsenic accumulation in rice grains. J. Hazard. Mater. 408 124913 (2021)

70. N. M. van Dam, M. Horn, M. Mares, I. T. Baldwin, Ontogeny constrains systemic protease inhibitor response in Nicotiana attenuata. J. Chem. Ecol. 27, 547-568 (2001).

71. T. Docimo et al., The first step in the biosynthesis of cocaine in Erythroxylum coca: The characterization of arginine and ornithine decarboxylases. Plant Mol. Biol. 78, 599-615 (2012)

72. R. A. R. Machado et al., Leaf-herbivore attack reduces carbon reserves and regrowth from the roots via jasmonate and auxin signaling. New Phytol. 200, 1234-1246 (2013)

73. M. L. Wong, J. F. Medrano, Real-time PCR for mRNA quantitation. Biotechniques 39 , 75-85 (2005)

74. F. Missirlis et al., Characterization of mitochondrial ferritin in Drosophila. Proc. Natl. Acad. Sci. U.S.A. 103, 5893-5898 (2006).

75. R. A. R. Machado, C. C. M. Arce, A. P. Ferrieri, I. T. Baldwin, M. Erb, Jasmonatedependent depletion of soluble sugars compromises plant resistance to Manduca sexta. New Phytol. 207, 91-105 (2015)

76. Y. Benjamini, Y. Hochberg, Controlling the false discovery rate: A practical and powerful approach to multiple testing. J. R. Stat. Soc. Series B Stat. Methodol. 57, 289-300 (1995).

77. M. R. Herve, RVAideMemoire: Testing and plotting procedures for biostatistics. https://cran.r-project.org/web/packages/RVAideMemoire/index.html. Accessed 28 June 2021.

78. J. Oksanen et al., Vegan: Community ecology package. R package version 2.0-10. CRAN.R-project.org/package vegan. Accessed 28 November 2020.

79. D. Bates, M. Machler, B. M. Bolker, S. C. Walker, Fitting linear mixed-effects models using Ime4. J. Stat. Softw. 67, 1-48 (2015).

80. R. V. Lenth, Least-squares means: The R Package Ismeans. J. Stat. Softw. 69, 1-33 (2016). 